\title{
Visual impressions of pushing and pulling: the object perceived as causal is not always the one that moves first
}

\author{
Peter A White \\ School of Psychology, Cardiff University, Tower Building, Park Place, Cardiff CF10 3AT, Wales, UK; \\ e-mail: whitepa@cardiff.ac.uk \\ Received 5 April 2012, in revised form 4 September 2012
}

\begin{abstract}
Stimuli were presented in which a moving object (A) contacted a stationary object (B), whereupon both objects moved back in the direction from which object A had come. When object B rapidly decelerated to a standstill, so that the two objects did not remain in contact, object $\mathrm{B}$ was perceived as pushing object $\mathrm{A}$. Thus, even though object $\mathrm{B}$ only moved when contacted by object $\mathrm{A}$, it was perceived as the causal object, as making something happen to object $\mathrm{A}$. This is contrary to the hypothesis that the object perceived as causal is always the object that moves first. It is, however, consistent with a theoretical account, in which visual causal impressions occur through a process in which visually picked-up kinematic information is matched to stored representations, based on experiences of actions on objects, which specify forces and causality as part of the perceptual interpretation of the event.
\end{abstract}

Keywords: phenomenal causality, launching effect, pushing impression, pulling impression

\section{Introduction}

It is well established that certain visual stimuli involving representations of objects in motion give rise to perceptual impressions that some kind of interaction is going on between the objects. The best known example of this is the launching effect. In a typical stimulus there are two objects, A and B, both simple two-dimensional geometrical forms such as rectangles or discs. Object B is initially stationary in the centre of the screen. Object A moves horizontally from left to right and comes into contact with object B. When contact occurs, object A stops moving and object B starts moving in the same direction at the same or slightly lesser speed. The stimulus is an animation and no causality is actually present. Observers of this stimulus, however, usually report a visual impression of causality: they see object A make object B move by striking it (Gordon et al 1990; Michotte 1963; Natsoulas 1961; Schlottmann et al 2006; Scholl and Tremoulet 2000). Studies of perceptual judgment and brain imaging studies support the contention that this is a genuine perceptual impression and not a mere product of higher cognition (Blakemore et al 2001; Choi and Scholl 2004, 2006; Fugelsang et al 2005; Newman et al 2008; Roser et al 2005; Scholl and Nakayama 2002, 2004). Developmental research indicates that young infants make perceptual discriminations that are consistent with the hypothesis that the launching effect occurs in their perceptual processing (Cohen and Amsel 1998; Cohen et al 1998; Cohen and Oakes 1993; Leslie and Keeble 1987; Muentener and Carey 2010; Newman et al 2008; Oakes 1994; Oakes and Cohen 1990; Saxe and Carey 2006).

The launching effect is not the only kind of visual causal impression that has been shown to occur: impressions of pushing or carrying, pulling, smashing to pieces, and penetrating have been reported (Michotte 1963; White and Milne 1997, 1999, 2003). However, all the kinds that have been identified so far share one feature: the object perceived as causal moves first. This is consistent with the possibility that perceived prior motion of the object perceived as causal is necessary for a visual causal impression to occur; more specifically, perceived prior motion of an object is necessary for it to be perceived as causing motion in another object. I shall refer to this as the "prior motion" hypothesis. 
Although the prior motion hypothesis has not been directly tested, no published evidence is inconsistent with it. There are at least two experiments demonstrating that a causal impression is eliminated when prior motion of what would otherwise be the causal object is absent. One of these is experiment 50 in Michotte (1963). Michotte arranged a moveable screen with a small disc positioned in front of the screen from the observer's point of view. Screen and disc started moving at the same time, with the same kinematic properties. Michotte reported that this is perceived as a single object beginning to move, with the disc perceived as part of this object. In experiment 52, everything was the same except that the screen began to move before the disc did. This time Michotte reported an impression that the screen entrained (ie carried) the disc. This fits with the prior motion hypothesis: in the absence of prior motion (of the screen, in this case), no causal impression occurred. The other relevant experiment is in White and Milne (1997). In this, five black squares were arranged in a column with gaps between them. The top object moved off horizontally at constant speed. After a short delay the second object moved off in the same direction at the same speed, and this continued until all five objects were in motion. Observers reliably reported an impression that the object that moved first was pulling the others, despite the fact that the objects never came into contact and had no visible connection. White and Milne presented stimuli in which all the objects began to move at the same time, and no impression of pulling was reported. This also fits with the prior motion hypothesis. Two studies have shown that the launching effect can occur when the visible objects are stationary (Gordon et al 1990; White 2005), but this is not inconsistent with the prior motion hypothesis because in both cases other features of the stimulus give rise to an impression of motion on the part of the causal object: there is perceived prior motion of the object identified as causal, even though the object is objectively not moving.

The necessity of perceived prior motion of the causal object is postulated in two different explanatory accounts of visual causal impressions. It is critical to Michotte's account. Michotte (1963) argued that visual impressions of causality occur because of certain structural properties of the stimuli. These structural properties satisfied Gestalt principles of perceptual grouping, so that the motions of the two objects were perceived as a single motion, that of the first-moving object which transferred itself to the other object, thereby bringing about its spatial displacement. In effect, the motion of object B is that of object A transferred to object B, and this accounts for the causal impression. In his summary of the theoretical account of the launching effect, Michotte (1963, page 143) wrote: "the essential point in our theory is that the movement of the motor object undergoes an 'extension'... on to the projectile". Michotte introduced the term "l'ampliation du mouvement" (rendered in English as "ampliation of the movement") and stated that this meant "the creating or establishing of the extension on to a second object of the already existing movement of a first object, in such a way that this movement brings about the displacement of the second object" (page 143, italics in original). Note the phrase, "already existing movement". Under Michotte's account, prior motion of the causal object is necessary for the launching effect to occur.

The same holds for Michotte's account of the entraining effect. This too was analysed as a case of ampliation of the movement. Michotte (1963, page 163) wrote: "The temporal priority of the movement of the motor object and the similarity of the movements of the two objects after their encounter constitute the essential conditions for the Entraining Effect". It is the latter, not the former, that differentiates entraining from launching. Michotte interpreted traction (the pulling impression) as a special case of entraining, differing only in the fact that the motor object takes up a position in front of the other object rather than behind it; prior motion of the motor object is still required for the causal impression to occur (Michotte 1963, pages 160-161). 
The second account that postulates the necessity of prior motion is the modular theory proposed by Leslie (1995) and called "ToBy". Leslie and Keeble (1987) proposed a module that would take input from lower-level processes of motion perception and generate a higherlevel description that would specify the causal structure of the event. Essentially, if the right kind of kinematic information is generated by lower-level processing, the module is brought into operation and generates a causal impression. The module hypothesis accounts for the rapid and automatic generation of the causal impression, for the impenetrability of the mechanism that generates it, and for its apparent immunity to influence from extrinsic processes (Scholl and Tremoulet 2000).

Leslie (1995) developed that account further by arguing that the causal impression involves a primitive notion of force, such that objects in motion possess or bear force and, when they contact other objects, they transmit force to those objects. Thus, in the launching effect, object A carries force and the production of motion in object B is accomplished by the transmission of force from object A to object B. Leslie argued that this understanding of the directionality of force accounts for the results of the study by Leslie and Keeble (1987). Prior motion of object A is critical to this account: "The event begins with object A moving and object B stationary. Therefore object A rather than object B has to be the initial bearer of force" (Leslie 1995, page 126). Leslie summarised the hypothesis as follows (1995, page 127): "The infant's 'theory of body' (ToBy) tacitly employs the idea that FORCE is transmitted from one object to another in a particular direction, from the moving to the stationary, like a baton being passed on in a relay race. There is no equal and opposite reaction as far as ToBy is concerned. This defines mechanical direction-the direction of FORCE transmission". In this account too, then, perceived prior motion for the unidirectional transmission of force is postulated in Leslie's account.

Leslie's account does not explain why an object in motion is understood as a bearer of force and a stationary object not. As Leslie (1995) pointed out, under Newton's third law of motion there is a symmetry of forces in a launching event, and it is as true to say that object B makes object A stop as to say that object A makes object B go. But this symmetry of forces is not perceived, and both causality and force transmission are perceived as unidirectional, from object A to object B (White 2006, 2007). Postulating an innate visual mechanism that makes this so is not a satisfactory explanation, because there could just as well have been an innate visual mechanism that would generate impressions that accorded with Newton's third law.

That problem is addressed in a different account of visual impressions of force and causality. In this account, kinematic information in the visual stimulus is matched to stored representations of interactions between objects. Additional features of the stored representations then form part of the perceptual interpretation of the stimulus. An early version of this idea was proposed by White and Milne (1997), and I have recently developed it further (White 2009, in press). The source of the stored representations is the actor's own actions on objects, comprising knowledge of motor output and haptic feedback. The haptic system is a mechanoreceptor system, and as such it yields impressions of forces being exerted in actions on objects. This gives rise to stored representations which integrate visually perceived information about kinematics with motor knowledge, and haptically perceived information about forces and causality. The impressions of forces are not guaranteed to be accurate: they are the end result of integrating haptic feedback with information about the intended action and consequences, and are therefore a kind of perceptual construction, drawing on information from within as well as what sensory input provides. Nonetheless, the haptic system is the only sensory system that detects contact forces, and is therefore the original source of any information about forces that finds its way into perception in other sensory modalities. 
The essential feature of this account is that causality is not detected in visual information directly. It is, instead, part of a perceptual interpretation made by matching kinematic information in visual input to kinematic information in stored representations, ultimately representations of actions on objects. The stored representations include visual, motor, and haptic information, so the motor and haptic information become part of the perceptual interpretation generated by the matching process. Critically, the stored representations do include information about causality, because causality is experienced through the combination of knowledge of motor output and haptic feedback (White 2009, 2012b). Thus, when a launching stimulus is presented, visual information about object kinematics is matched to stored representations of actions on objects; the motor/haptic component of these representations then becomes the impression of causality in the perceptual interpretation of the stimulus.

If the visually picked up kinematic information does not match any stored representations, at least approximately, or if it matches two (or more) different stored representations that have different mechanical properties, then no clear impression results. Take the example of two objects, A and B, initially in contact but stationary. Then both objects move off with the same kinematic properties, so that they remain in contact. The kinematics of this stimulus can be matched to stored representations of events in which A (or the actor) pushes B (the object acted on), to stored representations of events in which A (or the actor) pulls B, and to stored representations of events in which both objects generate motion and are conjoined. No clear perceptual impression should result.

Note that matching to stored representations that include information about causality is the sole determinant of whether a causal impression forms part of the perceptual interpretation of the stimulus or not. In fact, there are no absolute rules or criteria for determining what sort of causal impression will occur under which circumstances, other than those imposed by the laws of physics. This entails that prior motion of the object perceived as causal is not necessary for a causal impression to occur. If a match can be made to a stored representation in which the object identified as causal is not the one that moves first, a visual causal impression will occur in which the object that moves first is not identified as the causal object. To distinguish this account from those proposed by Michotte (1963) and Leslie (1995), therefore, it is simply necessary to demonstrate that a visual impression of causality can occur when the object perceptually identified as causal is not the one that moves first. In the present study I report evidence for such an impression.

Under what circumstance would an actual experience of causality occur in which the object moving first was not perceived as the causal object? One possibility concerns acts of interception or batting away of objects that are, or could be, on a collision course with the actor. In such cases, the actor's extremity would typically start to move before the object contacted it. For purposes of testing the prior motion hypothesis, it would be better to find an example in which the actor's extremity did not start to move until the object actually contacted it. As a simple example, a fly lands on one's hand, and one pushes the fly off with an outward thrust of the arm. The same can happen if a ball rolling over the floor contacts one's foot; the foot may then kick the ball away. The essential kinematic pattern of such interactions is that an object (A) moves to contact another, initially stationary object (B); object $\mathrm{B}$ rapidly accelerates in the opposite direction, and contact with object A is quickly lost as object B decelerates again, having imparted sufficient momentum to object A to expel it. This motion pattern is shown in figure 1, and is called, as neutrally as possible, the "separating motion" stimulus.

A perceptual interpretation of the separating motion stimulus in which object B is the cause would only occur if the kinematic features of the stimulus could be matched only to interactions of the kind exemplified by the fly and ball examples. Are there any other possibilities? 

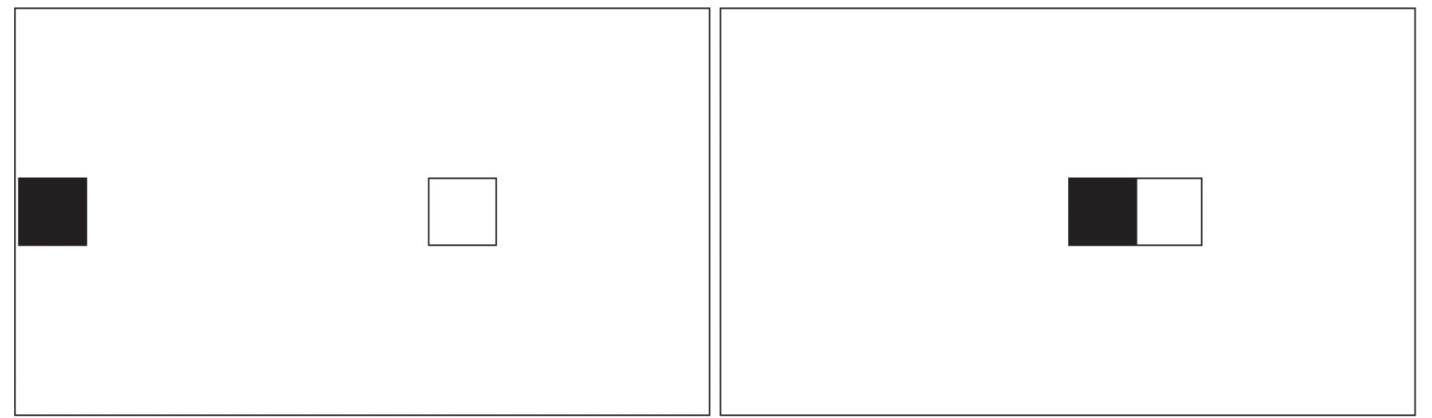

(a)

(b)

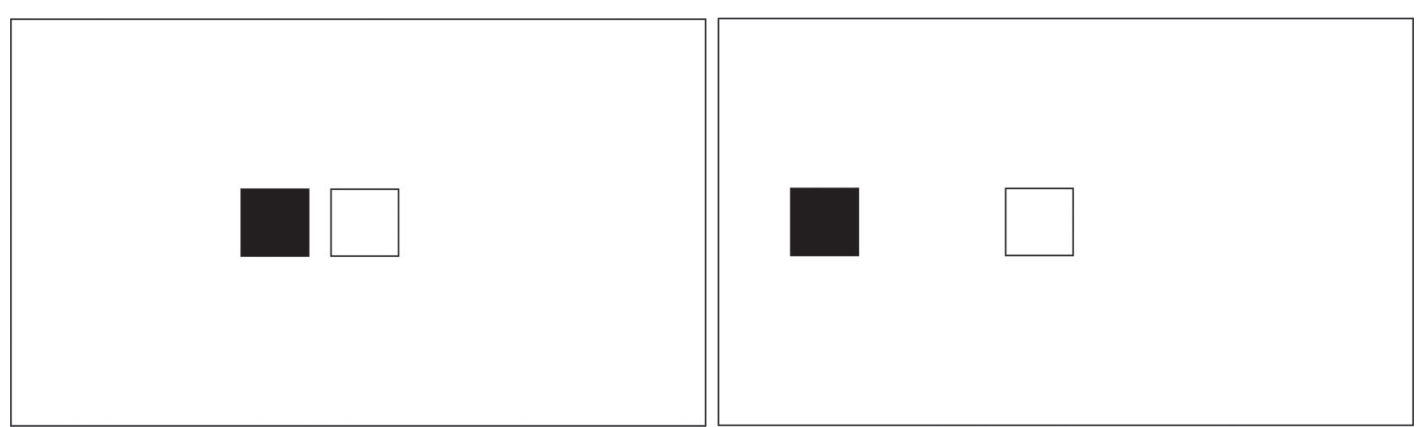

(c)

(d)

Figure 1. Separating motion stimulus. Figure 1a shows the first frame of the stimulus in which object A (the black square) is moving in from the left side of the frame towards object B (the white square), which is stationary towards the right side of the frame. Figure $1 \mathrm{~b}$ shows the frame in which object $\mathrm{A}$ contacts object $\mathrm{B}$. At the point shown in figure $1 \mathrm{~b}$ both objects start moving to the left. Object A moves at constant speed but object B decelerates. Figure 1c shows both objects in motion, but the gap between them is increasing as object B slows down. Figure 1d shows the frame in which object B comes to a halt. Object A continues to move in a straight line at constant speed until it exits the frame at the left side. Figure 1 shows slow deceleration; other stimuli have faster deceleration which brings objects B to a halt in fewer frames and close to its starting point.

One possibility drawn from the realm of human action is a reach-and-grasp motion. In this, object A would be the actor's hand, and object B would be an object grasped by the hand and pulled back towards the actor. The problem with this is that, in pulling actions, contact between hand and object is usually sustained, the whole point of the action being to hang on to the object. If the objects separate, as they do in the separating motion stimulus, that would be characteristic of a partial failure to grasp the object properly: the object is pulled a bit but then let go. Actions like that do happen, but they are rare. In the matching process, the perceptual interpretation is determined by majority features of matching stored representations, not by minority ones. I would contend, therefore, that the separating motion stimulus should be perceptually interpreted in such a way that object B is perceived as causing the post-contact motion of object A.

If the two objects remain in contact, a different interpretation applies. An example stimulus is shown in figure 2, which will be called, equally neutrally, the "shared motion" stimulus. This stimulus fits the features of a successful pulling action. Object A is the hand and grasps and pulls object B while retaining a grasp on it. It does not fit the kinematic pattern of batting an object away because, in such actions, contact between hand (or foot) and object is of brief duration. It is possible to imagine pushing actions that fit the kinematic pattern. For example, a parent places an object in an infant's hand and the infant, not interested, pushes it across a table-top to get rid of it, or even pushes the parent's hand away. Pushing with extended contact is, however, likely to be less common than pulling with extended contact. 


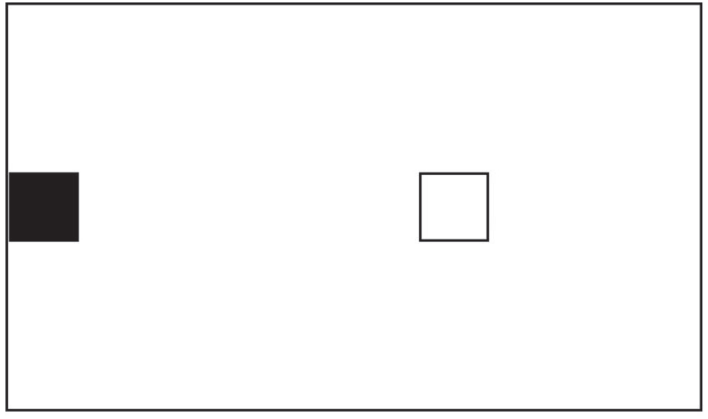

(a)

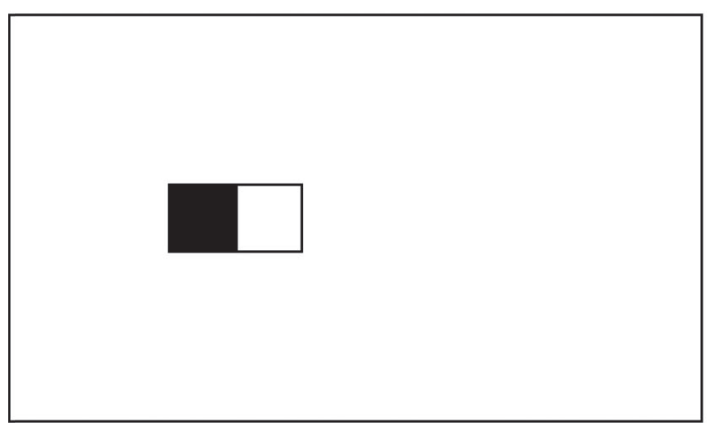

(c)

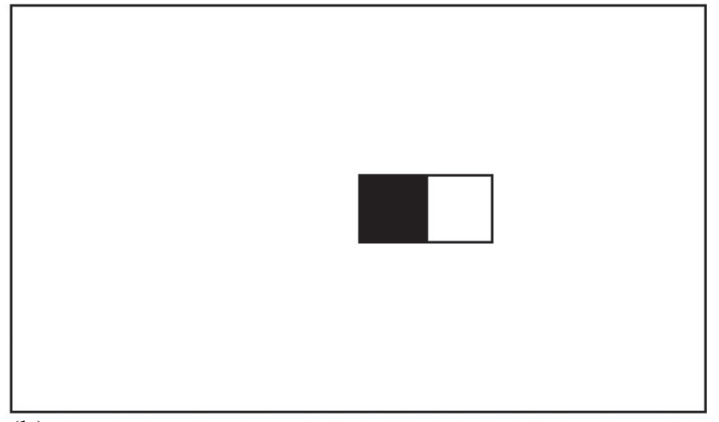

(b)

Figure 2. Shared motion stimulus. Figures 2a and $2 \mathrm{~b}$ are identical to figures $1 \mathrm{a}$ and $1 \mathrm{~b}$. At the point shown in figure $2 b$ both objects start moving towards the left at constant speed. Figure $2 \mathrm{c}$ shows a frame from that part of the stimulus. Motion continues in a straight line at constant speed until both objects exit the frame at the left side.

It is therefore likely that the shared motion stimulus will be perceived as a pulling stimulus with object A as the cause of object B's post-contact motion. This is less relevant to the prior motion hypothesis because, of course, object A, the object that should be perceived as causal, is the one that moves first. But it is important at least to show that it does not give rise to the same perceptual interpretation as the separating motion stimulus does.

To summarise, the matching account predicts that separating motion stimuli will tend to be perceived as pushing events; that is, events in which object B pushes object A; and shared motion stimuli will tend to be perceived as pulling events. Under the accounts proposed by Michotte (1963) and Leslie (1995), object A should be perceived as causal with both kinds of stimuli, because object A always moves first.

\section{Experiment 1}

This experiment was designed to ascertain whether the predicted phenomenon occurred, and to what extent it was modified by variations in kinematic features of the stimuli. Because I was not sure to what extent the impressions might be affected by kinematic variables, I created numerous stimuli conforming to the general features shown in figures 1 and 2, but varying in kinematic properties. In some stimuli the speed of object A prior to contact was constant; in others object A decelerated so that it was almost stationary at the point of contact with object B. The properties of motion after contact were varied as well. Stimuli fell into two distinct sets. Set 1 were all variations on the separating motion stimulus shown in figure 1. Set 2 were all variations on the shared motion stimulus shown in figure 2. Participants were presented with three brief verbal descriptions of pulling (A pulling B), pushing (B pushing $\mathrm{A}$ ), and independent motion, and chose the one that they felt best captured what they saw. It was predicted that stimuli in set 1 would be perceived as pushing stimuli (ie with object B as the causal object), and stimuli in set 2 would be perceived as pulling stimuli.

\subsection{Method}

2.1.1 Participants. Thirty first-year undergraduate students of psychology participated in return for course credit. All had normal or corrected-to-normal vision. 
2.1.2 Apparatus, stimuli, and design. Stimuli consisted of frame sequences generated by a Macintosh G3 computer and displayed on a Mitsubishi Diamond Plus 71 CRT colour monitor. The frames were presented in phase with the computer's vertical blank signal and appeared at the refresh rate of $67 \mathrm{~Hz}$. Each frame was 500 pixels $(18.5 \mathrm{~cm})$ wide by 300 pixels $(11.1 \mathrm{~cm})$ high. The boundaries of the frame appeared on the screen as thin black lines. These disappeared between stimulus presentations, leaving the screen uniform white. All sequences consisted of 200 frames, each lasting 2.7 s. The background of each frame was uniform white throughout. There were three sets of stimuli.

Set 1. Stimuli in this set were variations on the separating motion stimulus depicted in figure 1. Object B was stationary, a little to the right of the centre of the frame. Object A entered from the left and moved horizontally until it came into contact with object B. At that point both objects moved to the left but with different motion properties so that they did not remain in contact. The motion properties of object A prior to contact were manipulated with four values. In two of these, the speed of object A was constant at 8.1 or $18.9 \mathrm{~cm} \mathrm{~s}^{-1}$. In the other two, object A initially moved at one of the same two speeds but immediately began to decelerate. The rate of deceleration was constant, and was designed so that object A had almost come to a halt at the point where it contacted object $\mathrm{B}$. The rates of deceleration will be labelled as slow and rapid, respectively. The motion properties of object A after contact were manipulated with three values, constant speed at either $8.1 \mathrm{~cm} \mathrm{~s}^{-1}, 13.5 \mathrm{~cm} \mathrm{~s}^{-1}$, or $18.9 \mathrm{~cm} \mathrm{~s}^{-1}$. The motion properties of object B after contact were manipulated. Initially object B began to move at the same speed as object A, but deceleration started immediately so that the objects were in contact for only two frames of each stimulus. The rates of deceleration varied, depending on the initial speed, because they were designed to bring object $\mathrm{B}$ to a halt at the same spatial location. One rate of deceleration brought object B to a halt about one-third of the distance between the object's starting point and the left edge of the frame; this will be called slow deceleration. The other rate brought object $\mathrm{B}$ to a halt after a movement distance approximately equal to the length of a side of object B; this will be called rapid deceleration. These manipulations generated a total of 24 stimuli.

Set 2. Stimuli in this set were variations on the shared motion stimulus depicted in figure 2 . Object A moved to contact object B as in the separating motion stimulus. At that point both objects moved towards the left with the same motion properties, so that they remained in contact until they exited the frame on the left side. The motion properties of object A prior to contact were manipulated in the same way as in set 1: constant speed of 8.1 or $18.9 \mathrm{~cm} \mathrm{~s}^{-1}$, and the slow and rapid rates of deceleration. The motion properties of objects A and B after contact were manipulated with six values. In three of these the speed of the pair of objects was constant at either $8.1 \mathrm{~cm} \mathrm{~s}^{-1}, 13.5 \mathrm{~cm} \mathrm{~s}^{-1}$, or $18.9 \mathrm{~cm} \mathrm{~s}^{-1}$, similar to the post-contact motion of object $A$ in set 1 . In the other three, the initial speed of the pair was $2.7 \mathrm{~cm} \mathrm{~s}^{-1}$, and they immediately accelerated at one of three rates. Two of these were the inverse of the slow and rapid deceleration rates for object A prior to contact, and the third, labelled "medium", took an intermediate value. These manipulations also generated a total of 24 stimuli.

Other features of materials and design. An initial instruction sheet was used, with the following wording:

"In this experiment you will see a series of short movies, about one or two seconds in duration, each involving two objects. One object is a black square and the other object is a white square. In each movie one or both of the objects will be moving and at some point they will come into contact. Your task is to report what impression you have of what goes on after they come into contact. It might seem as though the black square pulls or carries the white square; it might seem as though the white square pushes or shoves the black square; or it might seem as though each object moves of 
its own accord and does not do anything to the other object. You just have to choose which of these three possibilities is closest to what you see.

So, for each movie, you will see the following three response options:

"It seems as though the black square pulls or carries the white square."

"It seems as though the white square pushes or shoves the black square."

"It seems as though each object moves of its own accord and does not do anything to the other object."

Just put a tick beside the one that is closest to what you see.

Please remember that we want you to record your immediate visual impression. You may find that you think about the movies in some way, but it isn't your thoughts that we want to know about. It is just your visual impression, how the movie looks to you. Don't worry if it seems a bit odd-just tell us what you see."

There was also a set of response sheets for the participant to record their choice for each stimulus. These identified each stimulus with the number of its order in the presentation sequence and included the three response options listed above.

The two sets added up to a total of 48 stimuli. These were presented in random order, and order of presentation was randomised independently for each participant.

2.1.3 Procedure. The experiment was run by an experimenter blind to the aims and hypotheses of the experiment. Participants were run individually in a small windowless laboratory, empty except for the equipment used for the experiment and with fluorescent lighting giving a low ambient light level. Participants were seated so that their faces were approximately $75 \mathrm{~cm}$ from the screen, and they were permitted to adjust this distance slightly for personal comfort. Participants first filled out an informed consent form. The experimenter introduced the experiment by giving the participant the written instructions.

The experimenter then told the participant that she would ask if they were ready. This was their cue to fixate the screen. When they said "yes", the first stimulus was presented twice. They then made their choice of response. This procedure was re-iterated until all the stimuli had been shown. Sessions lasted approximately $20 \mathrm{~min}$. At the end of the session the participant was thanked and given course credit and a debriefing sheet which explained the general aims of the research.

\subsection{Results}

Set 1. Endorsement frequencies for the pulling, pushing, and independent motion responses for set 1 are presented in table 1. The table shows that pushing was the preferred response for all stimuli, and was chosen by at least twenty-two of the thirty participants for every stimulus.

Table 1. Frequencies of endorsement of the three responses, experiment 1, set 1.

\begin{tabular}{|c|c|c|c|c|c|c|c|}
\hline \multirow[t]{2}{*}{ A before } & \multicolumn{6}{|c|}{$\mathrm{A}$ and $\mathrm{B}$ after } & \multirow[t]{2}{*}{ Mean } \\
\hline & $8.1 /$ slow & 8.1/rapid & $13.5 /$ slow & 13.5/rapid & 18.9/slow & 18.9/rapid & \\
\hline $8.1 \mathrm{~cm} \mathrm{~s}^{-1}$ & $4 / 24 / 2$ & $2 / 23 / 5$ & $4 / 25 / 1$ & $2 / 27 / 1$ & $2 / 27 / 1$ & $1 / 27 / 2$ & $2 / 25 / 2$ \\
\hline $18.9 \mathrm{~cm} \mathrm{~s}^{-1}$ & $7 / 22 / 1$ & $2 / 27 / 1$ & $5 / 24 / 1$ & $3 / 25 / 2$ & $3 / 26 / 1$ & $4 / 25 / 1$ & $4 / 25 / 1$ \\
\hline Slow & $4 / 23 / 3$ & $1 / 27 / 2$ & $0 / 29 / 1$ & $2 / 28 / 0$ & $1 / 28 / 1$ & $1 / 29 / 0$ & $1 / 27 / 1$ \\
\hline Rapid & $5 / 22 / 3$ & $3 / 24 / 3$ & $4 / 25 / 1$ & $3 / 26 / 1$ & $3 / 26 / 1$ & $4 / 25 / 1$ & $3 / 25 / 2$ \\
\hline Mean & $5 / 23 / 2$ & $2 / 25 / 3$ & $3 / 26 / 1$ & $2 / 26 / 1$ & $2 / 27 / 1$ & $2 / 26 / 1$ & $2 / 26 / 1$ \\
\hline
\end{tabular}


Endorsement data were analysed with the $\chi^{2}$ test. Since two options described different kinds of causal impression and the third described a lack of one, the assumption of equal likelihood across the options might not be justified. Accordingly, it was decided to analyse only the two most frequently endorsed options for each stimulus. In all 24 analyses a significant $(p<0.05)$ preference for the pushing option was found. It appears that, if object B does not remain in contact with object $\mathrm{A}$, then the event is reliably perceived as pushing, at least with the range of speeds and accelerations used in this experiment.

Set 2. Endorsement frequencies for the pulling, pushing, and independent motion responses for set 1 are presented in table 2. The table shows that pulling was the preferred response for all stimuli, and was chosen by more than half of the participants for all stimuli except two, where it was chosen by $50 \%$. Endorsement data were analysed with the $\chi^{2}$ test, in the same way as for set 1 . In 17 out of 24 stimuli these two were the two causal options, and in the remainder they were the pulling and independent motion options. Significant $(p<0.05)$ $\chi^{2}$ values were found in 21 of the 24 analyses, and in all cases the pulling option was the preferred response.

Table 2. Frequencies of endorsement of the three responses, experiment 1, set 2.

\begin{tabular}{|c|c|c|c|c|c|c|c|}
\hline \multirow[t]{2}{*}{ A before } & \multicolumn{6}{|c|}{$\mathrm{A}$ and $\mathrm{B}$ after } & \multirow[t]{2}{*}{ Mean } \\
\hline & $8.1 \mathrm{~cm} \mathrm{~s}^{-1}$ & $13.5 \mathrm{~cm} \mathrm{~s}^{-1}$ & $18.9 \mathrm{~cm} \mathrm{~s}^{-1}$ & slow & medium & rapid & \\
\hline $8.1 \mathrm{~cm} \mathrm{~s}^{-1}$ & $19 / 5 / 6$ & $20 / 6 / 4$ & $23 / 5 / 2$ & $15 / 8 / 7$ & $21 / 5 / 4$ & $22 / 8 / 0$ & $20 / 6 / 4$ \\
\hline $18.9 \mathrm{~cm} \mathrm{~s}^{-1}$ & $20 / 6 / 4$ & $22 / 5 / 3$ & $22 / 6 / 2$ & $20 / 7 / 3$ & $20 / 6 / 4$ & $24 / 5 / 1$ & $21 / 5 / 3$ \\
\hline Slow & $18 / 3 / 9$ & $19 / 8 / 3$ & $15 / 14 / 1$ & $18 / 4 / 8$ & $19 / 7 / 4$ & $21 / 6 / 3$ & $18 / 7 / 5$ \\
\hline Rapid & $21 / 5 / 4$ & $22 / 6 / 2$ & $22 / 6 / 2$ & $22 / 3 / 5$ & $22 / 2 / 6$ & $27 / 2 / 1$ & $22 / 4 / 3$ \\
\hline Mean & $19 / 5 / 6$ & $21 / 6 / 3$ & $20 / 8 / 2$ & $19 / 5 / 6$ & $20 / 5 / 4$ & $23 / 5 / 1$ & $20 / 5 / 4$ \\
\hline
\end{tabular}

Note. Response frequencies are presented in the order pulling/pushing/independent motion. "Slow", "medium", and "rapid" refer to rates of deceleration in the motion of object A prior to contact (A before) and to rates of acceleration in the motion of objects $\mathrm{A}$ and $\mathrm{B}$ after contact (A and B after).

\subsection{Discussion}

The results were as predicted under the matching hypothesis. In set 1, objects A and B separated after contact occurred because of the deceleration of object B; and pushing, with object $\mathrm{B}$ as the causal object, was consistently the preferred response. In set 2, motion properties were similar to those in set 1 except that the two objects remained in contact, and pulling was reliably the preferred response.

One possible problem with experiment 1 is that the three response options could suggest possible interpretations of the stimuli that would not otherwise have occurred to participants. This would not be sufficient to explain the observed preferences, because the mere presence of three response options does not direct participants to any particular one of them. However, if a stimulus is perceptually ambiguous, the response options could suggest ways of disambiguating the stimuli, perhaps in higher cognition. Participants might even be able to think about resemblances between the stimuli and various kinds of interactions. Ideally it should be possible to show that a pushing impression occurs for a set 1 stimulus, even when participants are not asked about their causal impressions. Experiment 2 was designed to do that. 


\section{Experiment 2}

The method for this experiment was modelled on a study carried out by Schlottmann et al (2006). They used a free verbal response method in which a series of stimuli was presented and participants wrote down a description of what they saw for each stimulus. They presented 12 stimuli. In this experiment, 10 stimuli were presented, 4 drawn from sets 1 and 2 of experiment 1 , and the other 6 functioning as control/filler stimuli.

\subsection{Method}

3.1.1 Participants. Forty first-year undergraduate students of psychology participated in return for course credit. All had normal or corrected-to-normal vision. None had participated in experiment 1 and none had been taught any psychology of relevance to this research.

3.1.2 Apparatus, stimuli, and materials. Details of apparatus were as for experiment 1. 10 stimuli were constructed; each is described below with an identifying label. The first 4 are the key stimuli for the experiment.

1. Separating motion 1. This was the stimulus from experiment 1 , set 1 , with slow deceleration of object A prior to contact, object A moving at $18.9 \mathrm{~cm} \mathrm{~s}^{-1}$ after contact, and slow deceleration for object B.

2. Separating motion 2. This was similar to separating motion 1 except that there was rapid deceleration for object $\mathrm{B}$ after contact.

3. Shared motion 1. This was the stimulus from experiment 1 , set 2 , with rapid deceleration of object A prior to contact, initial post-contact speed of $2.7 \mathrm{~cm} \mathrm{~s}^{-1}$ for both objects, and slow acceleration of both objects, which remained in contact.

4. Shared motion 2. This was similar to shared motion 1 except that there was rapid acceleration of both objects after contact.

5. Launching. This was a standard launching stimulus similar to those used in previous experiments (Michotte 1963; Natsoulas 1961; Schlottmann et al 2006). Object A entered from the left as in the separating and shared motion stimuli, moving at a constant speed of $16.2 \mathrm{~cm}^{-1}$. On contact with object B, object A stopped and object B started moving to the right at a constant speed of $13.5 \mathrm{~cm} \mathrm{~s}^{-1}$. Natsoulas reported that the launching effect was strongest when object B's speed was slightly slower than that of object A, and that is why the two objects in this stimulus had different speeds.

6. Entraining. This was similar to entraining stimuli used by Michotte (1963). It was similar to the launching stimulus, except that, when object A contacted object B, both objects moved to the right at a constant speed of $13.5 \mathrm{~cm} \mathrm{~s}^{-1}$ and remained in contact. This kind of stimulus reliably gives rise to a visual impression that object $\mathrm{A}$ is pushing object $\mathrm{B}$ (Michotte 1963).

7. Right angle. This was similar to the launching stimulus, except that, when object $\mathrm{A}$ contacted object B, object B moved vertically upwards at $13.5 \mathrm{~cm} \mathrm{~s}^{-1}$. With this kind of stimulus, the launching effect is usually weak or absent (Michotte 1963; White 2012a).

8. Gap. This was similar to the launching stimulus, except that object A stopped before reaching object $\mathrm{B}$. The gap between the two objects was equal to twice the width of one of them. As soon as object A stopped, object B started moving as in the launching stimulus. With this kind of stimulus, the launching effect is usually weakened, though this depends on the speeds of the objects (Michotte 1963; Yela 1952).

9. Enforced disintegration. This was based on stimuli used by White and Milne (1999). The stimulus resembles a launching stimulus, except that, when object A contacts object B, object B disintegrates into 9 small rectangles that disperse in various directions, though with a rightward tendency. To maintain consistency with the other stimuli for this experiment, object A was a black rectangle instead of the black disc used by White and Milne (1999). 
Object B was also a rectangle composed of 9 smaller rectangles with spaces of 1 pixel between them (this was a software requirement). When object $\mathrm{A}$ contacted object $\mathrm{B}$, object $\mathrm{A}$ continued to move to the right at $2.7 \mathrm{~cm} \mathrm{~s}^{-1}$ and the 9 small rectangles dispersed at $13.5 \mathrm{~cm} \mathrm{~s}^{-1}$. Motion continued until all 9 rectangles has exited the frame. This stimulus usually gives rise to an impression that object A smashes object B to pieces (White and Milne 1999).

10. Shattering. This was based on stimuli used by White and Milne (1999). The rectangle composed of 9 small rectangles in the enforced disintegration stimulus took the part of object A. It entered from the left and moved towards a large rectangle (object B) that was stationary near the right side of the frame. Figure 3 a shows the first frame of the stimulus with object A entering from the left at $16.2 \mathrm{~cm} \mathrm{~s}^{-1}$. Figure $3 \mathrm{~b}$ shows the frame in which object A contacts object B. At that point the 9 small rectangles disperse in different directions with a leftward tendency, at $13.5 \mathrm{~cm} \mathrm{~s}^{-1}$. Figure $3 \mathrm{c}$ shows an early stage in the dispersal and figure $3 \mathrm{~d}$ shows a later stage. Motion continued until all rectangles had exited the frame. This stimulus usually gives rise to an impression that object $\mathrm{A}$ is shattered to pieces by its contact with object B (White and Milne 1999).



(a)

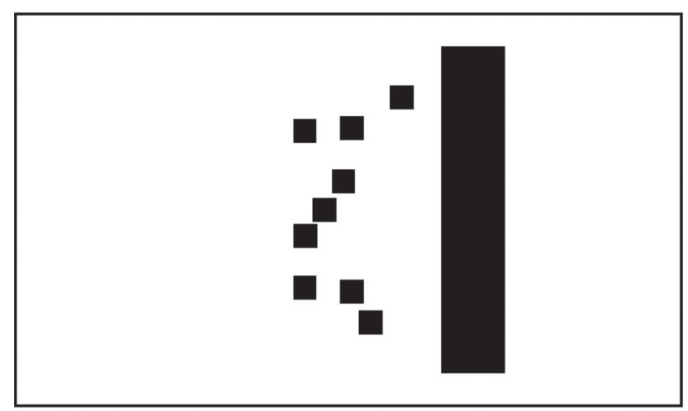

(c)

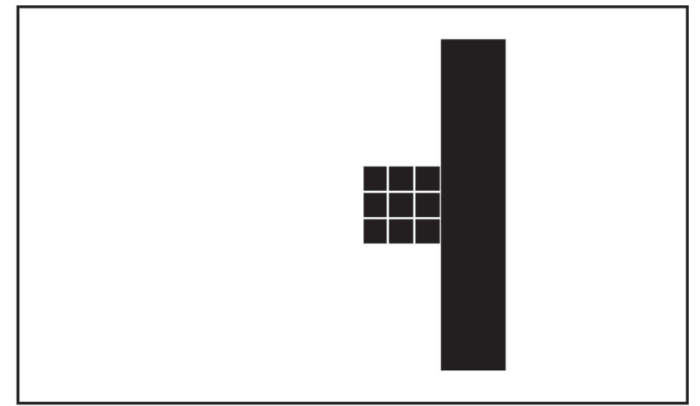

(b)

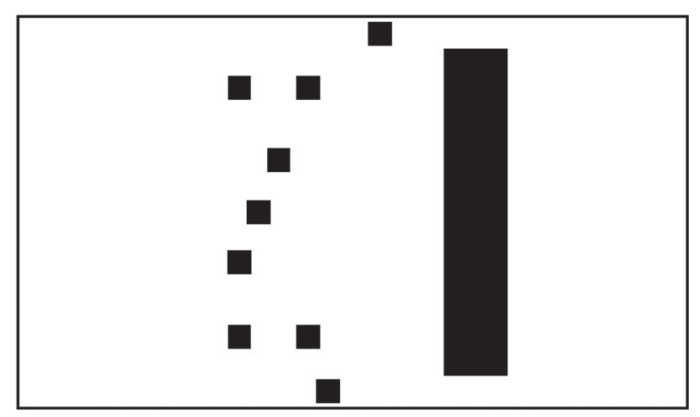

(d)

Figure 3. Shattering stimulus, based on White and Milne (1999). Figure 3a shows the first frame of the stimulus, with object A entering from the left and object B stationary near the right side. Figure $3 \mathrm{~b}$ shows the frame in which object A contacts object B. Figure 3c shows the component rectangles of object A dispersing after contact with object B, which remains stationary. Figure $3 \mathrm{~d}$ shows a later stage in the dispersal of the small rectangles.

The participant was presented with a booklet for his or her responses. On the front page of the booklet were the following instructions, the first paragraph of which is taken from Schlottmann et al (2006).

"This study is about how people perceive events involving moving objects. Your task is simply to describe what is happening in these events and what these objects are doing. We are interested in your subjective, intuitive impressions. Use everyday language, like you would to describe the event to a friend. Use a new page for each event and do not go back to look at your previous descriptions. 
You will see each event once. If you wish to see it again, please ask me.

When you have finished reading this, let me know that you are ready. I will then present the first event. When you have finished writing about that event, let me know and I will present the next one. This will continue until all the events have been presented."

Following that were 10 pages headed "Event 1", "Event 2", and so on, but blank apart from that. Participants wrote their descriptions on those pages.

3.1.3 Procedure. The procedure was as for experiment 1, except that each stimulus was presented once, and the experimenter ensured that the participant turned over the page after completing his or her description and before the next stimulus was shown. Order of presentation of stimuli was randomised independently for each participant.

\subsection{Results}

To code the descriptions, two objective linguistic criteria were used to identify causal interpretations. One was the use of a transitive verb with one of the objects as the grammatical subject, and the other as the grammatical object, and with an explicit outcome for the latter object. Examples would be, "[Object A] smashed [object B] to pieces" (enforced distintegration stimulus), and "[Object A] pushed [object B] to right" (entraining stimulus). The other criterion was use of the word "cause" and its derivatives to describe the interaction, specifically as one of the objects causing something to happen to the other one. Examples would be: "[Object A] hit [object B], causing each block to move off in a different direction" (enforced disintegration stimulus), and "[Object A] hit [object B] which caused [object B] to move as [object A] was moving" (entraining stimulus).

This is a stricter criterion than that used by Schlottmann et al (2006) for causality. They coded a statement as causal "if it used a verb with a causal component" (page 120), but one of the examples they gave was "hit". That is not sufficient to establish a description of a causal relation because there is no explicit outcome for object B. A moving object could hit a wall and stop or rebound without doing anything perceptible to the wall, and this would not be a causal relation. Similarly, grammatical constructions in which object A is described as contacting object $\mathrm{B}$ and object $\mathrm{B}$ is then described as doing something do not suffice to establish a description of a causal relation because it is not clear that the outcome for object $\mathrm{B}$ was perceived as caused by object A, and not by object B itself. An example would be: "[Object A] collides with [object B], [object B] shoots upwards quickly" (right angle stimulus). These were coded as "succession" descriptions.

The main interest in this experiment lies in which of the objects tends to be identified as causal for each stimulus. Table 3 shows the numbers of participants out of forty identifying each object as causal for each stimulus; in addition it shows numbers using succession descriptions, and numbers using terms appropriate for animate beings.

As table 3 shows, for both of the separating motion stimuli, a majority of participants provided descriptions in which object B was identified as causal. For separating motion 1, the most common term used was "pushed" (twelve participants), with other terms including "nudged" (one), "hit away (or out of screen/frame)" (six), "repelled" (two), "forced back" (two), "sent [object A] to left" (one), and expressions including the word "cause" or a derivative (three). For separating motion 2, the terms used included "pushed" (twelve), "made [object A] change direction and go back" (one), "hit away" (eight), "bumped off screen" (one), "nudged" (two), "sends [object A] moving to left" (one), "repelled" (one), "makes [object A] go in opposite direction" (one), and one expression including the word "cause". In fact, more participants identified object B as causal for the separating motion stimuli than identified object A as causal for the launching and entraining stimuli, although the differences were not statistically significant $\left(\chi^{2}=2.58\right.$ for the comparison between separating motion 2 and launching). 
Table 3. Numbers of participants identifying each object as causal, producing temporal succession and animate descriptions for each stimulus, experiment 2.

\begin{tabular}{lcccl}
\hline Stimulus & Object A & Object B & Succession & Animacy \\
\hline Separating motion 1 & 0 & 26 & 6 & 2 \\
Separating motion 2 & 1 & 28 & 3 & 1 \\
Shared motion 1 & 3 & 9 & 28 & 0 \\
Shared motion 1 & 3 & 7 & 29 & 1 \\
Launching & 21 & 0 & 15 & 0 \\
Entraining & 23 & 0 & 15 & 1 \\
Right angle & 9 & 0 & 21 & 0 \\
Gap & 3 & 0 & 0 & 3 \\
Enforced disintegration & 18 & 0 & 21 & 0 \\
Shattering & 0 & 0 & 35 & 0 \\
\hline
\end{tabular}

For the shared motion stimuli, few participants made an unambiguous causal identification, and a majority of those identified object B as causal, although the difference between those identifying object B and those identifying object A was not statistically significant $\left(\chi^{2}=3.00\right.$ for shared motion 1$)$. Most participants used descriptions of temporal succession, and almost all of those described object A as hitting or contacting object B and then both objects moving together (twenty-seven for shared motion 1 and twenty-nine for shared motion 2).

No participant identified object B as causal for any stimulus other than the shared and separating motion stimuli. As expected, more participants identified object A as causal for the launching and entraining stimuli than for the right angle and gap stimuli, with all differences being statistically significant by $\chi^{2}$ test. A substantial number identified object A as causal for the enforced disintegration stimulus as well, supporting the findings of White and Milne (1999) for that stimulus. No clear causal identifications were made for the shattering stimulus. Five participants identified the impact between object A and object B as the cause of the disintegration of object $A$; the remainder provided descriptions of the general form that object $\mathrm{A}$ hit object $\mathrm{B}$ and then broke into pieces.

Table 3 shows that descriptions of temporal succession were common for most of the stimuli. Overt descriptions in terms of animacy were rare, however. For the separating motion stimuli, the animate descriptions were: "[Object B] snapped at [object A]", "[Object B] appeared to be aggressive", and "[Object B] scared off [object A]". Each one therefore identified object $B$ as animate and causal. These are not included in the count of descriptions identifying object B as causal in table 3 .

One final observation is that several descriptions included explicit mention of force or equivalent terms. For example, for separating motion 1 there were 6 such descriptions, including "[Object B] hits [object A] back with a large force", and "[object A] was violently pushed away by [object B]". There were another 6 such descriptions for the shattering stimulus, including "[Object A] bashes into [object B] and shatters", and "[Object A] hit [object B] with force and shattered". Such descriptions were less common for the other stimuli, and there was none for the launching stimulus. These descriptions support the basic contention of White $(2007,2009)$ that impressions of force do occur in visual perception of interactions between objects; they are perhaps more noticeable, or only spontaneously described, when the amount of force involved appears unusually great. 


\subsection{Discussion}

The instructions and other materials for this experiment made no mention of causality, nor gave any indication of what sort of description was sought. It would be easy to provide descriptions merely of the kinematics of the stimuli, as a few participants did, but the majority produced spontaneous causal descriptions for at least some of the stimuli, and table 3 shows that there were consistent tendencies in the kinds of causal descriptions produced. It appears to be quite natural to describe these stimuli in terms of causal relations without prompting.

Specifically, the results for the separating motion stimuli confirmed those of experiment 1 . A high proportion of participants wrote accounts in which object $\mathrm{B}$ was described as causal in relation to object $A$, using terms semantically related to the notion of pushing. There was only one description identifying object $A$ as causal (for separating motion 2): this was, "[Object A] hit [object B] which caused [object B] to bounce back". As there was nothing in the materials that would prompt participants with the idea of a pushing event, it is likely that most participants really had a causal impression in which object B pushed object A.

The results for the shared motion stimulus did not confirm those of experiment 1 . Descriptions of object A pulling object B were rare, and outnumbered by descriptions in which object B pushed object A. The majority, however, described the two objects as moving together after contact, with causality not explicitly ascribed to either one. This might help to explain the disparity with the results of experiment 1 , because joint motion was not one of the available response options in that experiment. On the other hand, it is not clear why the absence of that option would lead participants to endorse the pulling descriptor in preference to the pushing descriptor. One possibility is that participants might have been sensitised, by the large number of similar stimuli, to the contrast between the separating motion and shared motion stimuli. That might have pushed them in the direction of choosing the contrasting description for the shared motion stimuli. That contrast would not have been apparent in the present experiment because there were only two stimuli of each kind and they were embedded in a context of other kinds of stimuli.

Schlottmann et al (2006) reported 92\% causal descriptions for both launching and entraining stimuli. The corresponding percentages here were lower, $52 \%$ for launching and $57 \%$ for entraining. One possible explanation for this is that a stricter linguistic criterion was used in the present experiment. Schlottmann et al (2006) coded descriptions of object A as "hitting" object B as causal, whereas that would not have been coded as causal in the present study unless there was a described outcome for object B. The lower percentage is not necessarily indicative of the actual proportion who had a causal impression on seeing the stimulus. Participants might have had a causal impression but chosen not to include it in their descriptions, and focussed on the kinematics instead. A substantial number of accounts described mere temporal succession: fifteen participants offered variations on "Object A hits (bumps into) object B and object B moves off (shoots off) the screen". These were not counted as causal descriptions, because causality is not explicit in them. It is quite possible, however, that participants had a causal impression and just chose the language of mere temporal succession to describe it. It is likely, therefore, that the true rate of occurrence of causal impressions is higher than the data in table 3 would suggest, and that they were either not included or not clearly expressed in the verbal responses.

The shattering stimulus provides an informative counterpoint to the separating motion stimuli. It shares with them the feature that object $\mathrm{A}$ is in motion prior to contact and reverses direction after contact (albeit disintegrating in doing so). There were, however, no descriptions in which object B was identified as causal. The key difference is that object B was stationary throughout the shattering stimulus, but moved on contact in the separating motion stimulus. 
It is likely that, in a version of the separating motion stimulus in which object B remained stationary throughout, object A would have been perceived merely as rebounding off object $\mathrm{B}$. Probably, therefore, some motion or activity in an object is necessary for that object to be perceived as causal. The results show, however, that the object perceived as causal does not have to be the one that moves first, and in fact does not have to move at all prior to being contacted by the other object.

\section{Experiment 3}

The results of experiment 1 show a strong tendency to endorse the pushing response with the separating motion stimuli, but they shed little light on the way in which this reported impression is influenced by other kinematic variables. Experiment 3 was therefore designed with a rating scale measure, thereby permitting a closer examination of stimulus variables in a parametric design. The motion properties of object A before contact and of object B after contact were manipulated.

\subsection{Method}

4.1.1 Participants. Thirty-two first-year undergraduate students of psychology participated in return for course credit. All had normal or corrected-to-normal vision. None had participated in the previous experiments.

4.1.2 Apparatus, stimuli, design, and procedure. General features of apparatus and stimuli were as in experiment 1 . In all stimuli object B was stationary prior to contact and object A moved from right to left after contact (ie reversing direction) at a constant speed of $18.9 \mathrm{~cm} \mathrm{~s}^{-1}$.

Two variables were manipulated. Motion of object A prior to contact was manipulated with four values: constant speed of $8.1 \mathrm{~cm} \mathrm{~s}^{-1}$; initial speed of $8.1 \mathrm{~cm} \mathrm{~s}^{-1}$ and decelerating at such a rate that it was almost stationary at the point of contact with object $\mathrm{B}$; constant speed of $18.9 \mathrm{~cm} \mathrm{~s}^{-1}$; and initial speed of $18.9 \mathrm{~cm} \mathrm{~s}^{-1}$ and decelerating at such a rate that it was almost stationary at the point of contact with object B. These were treated as four levels of a single independent variable and are identified as slow/constant, slow/decelerating, fast/constant, and fast/decelerating, respectively. It could be argued that these are two variables each with two values, but they are not because the rate of deceleration differs between the slow/decelerating and fast/decelerating conditions. Motion of object B after contact was manipulated with four values: constant speed of $8.1 \mathrm{~cm} \mathrm{~s}^{-1}$; constant speed of $18.9 \mathrm{~cm} \mathrm{~s}^{-1}$; initial speed of $18.9 \mathrm{~cm} \mathrm{~s}^{-1}$ with the slow deceleration rate from set 1 of experiment 1 ; and initial speed of $18.9 \mathrm{~cm} \mathrm{~s}^{-1}$ with the fast deceleration rate from set 1 of experiment 1 . These were treated as four levels of a single independent variable and are identified as slow/constant, fast/constant, fast/slow, and fast/fast, respectively. Orthogonal manipulation of the two variables yielded 16 stimuli. These were presented in random order, and order of presentation was randomised independently for each participant.

These manipulations replicate some of the stimuli from experiment 1. Stimuli where object B decelerates from its initial speed after contact conform to some of those in set 1, which reliably gave rise to endorsement of the pushing response. Stimuli where object B maintains a constant speed of $18.9 \mathrm{~cm} \mathrm{~s}^{-1}$ after contact, and therefore remains in contact with object A, conform to some of those in set 2, most of which gave rise to endorsement of the pulling response. Stimuli in which object B maintains a constant speed of $8.1 \mathrm{~cm} \mathrm{~s}^{-1}$ after contact do not precisely resemble any that were used in experiment 1 .

Initial written instructions to participants read as follows:

"In this experiment you will see a series of short movies, about one or two seconds in duration, each involving two objects. One object is a black square and the other object is a white square. In each movie one or both of the objects will be moving and at some point they will come into contact. Your task is to report what impression you 
have of what goes on after they come into contact. It might seem as though the black square pulls or carries the white square; it might seem as though the white square pushes or shoves the black square; or it might seem as though each object moves of its own accord and does not do anything to the other object.

To report your impression of what you see, please write a rating between -100 and +100 . The numbers have the following meanings:

-100 means that you had a very strong impression that the black square pulls or carries the white square.

0 (zero) means that you had no impression of pulling or pushing and it just seemed as though each object moved of its own accord.

+100 means that you had a very strong impression that the white square pushes or shoves the black square.

The stronger the impression you had that the black square pulls or carries the white square, the lower the number below zero you should put. The stronger the impression you had that the white square pushes or shoves the black square, the higher the number above zero you should put. You can write any number between -100 and +100 , whichever best expresses the impression you had.

Please remember that we want you to record your immediate visual impression. You may find that you think about the movies in some way, but it isn't your thoughts that we want to know about. It is just your visual impression, how the movie looks to you. Don't worry if it seems a bit odd - just tell us what you see."

There was also a set of response sheets for the participant to make his or her rating for each stimulus.

The procedure was similar to that for experiment 1 .

\subsection{Results}

Data were analysed with a 2 within (object A before, slow/constant vs slow/decelerating vs fast/constant vs fast/decelerating) $\times 2$ within (object B after, slow/constant vs fast/constant vs fast/slow vs fast/fast) analysis of variance (ANOVA). Means are reported in table 4 . The main feature of this table is that mean judgments were consistently positive for stimuli in which object B decelerated from its initial post-contact speed. The other stimuli did not give rise to strong impressions of either pushing or pulling.

There was a significant effect of object B after $\left(F_{3,93}=12.26, \mathrm{MSE}=5263.25, p<0.001\right)$. A posteriori paired comparisons were carried out with the Tukey test. The results, summarised in table 4, show that the stimuli in which object B decelerated from its initial post-contact speed received higher ratings than the other stimuli.

The main effect of object A before was not significant $\left(F_{3,93}=1.01, \mathrm{MSE}=1410.03\right)$. However, there was a significant interaction between the two variables $\left(F_{9,279}=3.71\right.$, $\mathrm{MSE}=1457.35, p<0.001)$. The interaction is depicted in table 4.

Starting with effects of object A before, at object B after slow/constant $\left(F_{3,93}=1.43\right.$, $\mathrm{MSE}=816.82$, not significant $)$; at object $\mathrm{B}$ before fast $/$ constant $\left(F_{3,93}=2.24, \mathrm{MSE}=316.72\right.$, not significant $)$; at fast $/$ slow $\left(F_{3,93}=6.17, \mathrm{MSE}=1078.05, p<0.001\right)$; at fast $/$ fast $\left(F_{3,93}=3.80\right.$, $\mathrm{MSE}=721.49, p<0.05)$. Both of these significant results show a similar pattern, with ratings being highest (ie towards the pushing end of the scale) when object A moved at a constant speed of $8.1 \mathrm{~cm} \mathrm{~s}^{-1}$ prior to contact, and lower at the other values of object A before.

Turning to effects of object $\mathrm{B}$ after, at object $\mathrm{A}$ before slow/constant $\left(F_{3,93}=22.07\right.$, $\mathrm{MSE}=2173.36, p<0.001)$; at slow/decelerating $\left(F_{3,93}=4.45, \mathrm{MSE}=2554.32, p<0.01\right)$; at fast $/$ constant $\left(F_{3,93}=6.35, \mathrm{MSE}=2697.99, p<0.001\right)$. These three significant effects all resemble the trend in the main effect, with higher means when object B decelerated from its initial post-contact speed than when it did not. At fast/decelerating, however, the effect was 
Table 4. Mean ratings of pulling/pushing, experiment 3.

\begin{tabular}{llllll}
\hline Object A before & \multicolumn{2}{l}{ Object B after } & \multicolumn{2}{l}{ All } \\
\cline { 2 - 4 } & slow/constant & fast/constant & fast/slow & fast/fast & \\
\hline Slow/constant & 11.50 & -14.84 & 65.44 & 59.81 & 30.48 \\
Slow/decelerating & 24.59 & 0.97 & 32.03 & 46.03 & 25.91 \\
Fast/constant & 12.72 & -4.03 & 45.16 & 39.72 & 23.96 \\
Fast/decelerating & 18.75 & 20.72 & 38.81 & 40.69 & 29.74 \\
All & $16.89^{\mathrm{a}}$ & $0.70^{\mathrm{a}}$ & $45.36^{\mathrm{b}}$ & $46.56^{\mathrm{b}}$ &
\end{tabular}

Note. Ratings are on a scale from -100 (pulling) to +100 (pushing). In the bottom row, means not sharing the same superscript differ at $p<0.05$ (Tukey).

not found $\left(F_{3,93}=1.95, \mathrm{MSE}=2209.62\right)$. The means indicate a trend in the same direction as the main effect, but it was not statistically significant.

\subsection{Discussion}

The principal findings confirm those reported in experiments 1 and 2, that an impression of object B pushing object A occurs consistently for separating motion stimuli where object B rapidly decelerates. This was found for all kinds of motion of object A prior to contact, though it was most evident when the speed of object A prior to contact was constant at its slower value, $8.1 \mathrm{~cm} \mathrm{~s}^{-1}$. The difference between this and the other stimuli was not great and perhaps requires confirmation in a further study.

No stimulus in this study gave rise to a consistent impression of pulling. The stimuli where objects A and B remain in contact and move from right to left together are the four fast/constant stimuli. The means for these cluster around zero, and in this respect the findings resemble those of experiment 2 more than those of experiment 1 . It appears that stimuli of that sort are genuinely ambiguous. There is sufficient evidence of a pulling impression from other research (Michotte 1963; White 2010; White and Milne 1997) to suggest that the failure to find it in the present experiment was due to specific features of the stimuli. One possibility is that object A's post-contact speed was too fast, and that pulling is an impression that emerges more distinctly at slower post-contact speeds. White and Milne (1997) found that the pulling impression was weaker at higher speeds, and the highest speed used in that study was $13.7 \mathrm{~cm} \mathrm{~s}^{-1}$, slower than the $18.9 \mathrm{~cm} \mathrm{~s}^{-1}$ used in experiment 3 . Speeds used in the study by White (2010) were lower still, $5.4 \mathrm{~cm} \mathrm{~s}^{-1}$ and $8.1 \mathrm{~cm} \mathrm{~s}^{-1}$. Experiment 4 was designed to test this possibility.

\section{Experiment 4}

\subsection{Method}

The method was similar to that of experiment 3 except for the following features. There were thirty participants, none of whom had participated in the previous experiments. Three variables were manipulated. Motion of object A prior to contact was manipulated with two of the values used in experiment 3, slow/constant and slow/decelerating. Speed of object A after contact was manipulated with two values, $8.1 \mathrm{~cm} \mathrm{~s}^{-1}$ ("slow") and $18.9 \mathrm{~cm} \mathrm{~s}^{-1}$ ("fast"), the latter being the speed used in experiment 3. Motion of object B after contact was manipulated with three values. In each case the initial speed was $8.1 \mathrm{~cm} \mathrm{~s}^{-1}$. The three values were constant (no deceleration), so that object B remained in contact with object A at its slow speed but not at its fast speed, and slow and fast rates of deceleration. These rates were chosen to bring object $\mathrm{B}$ to a halt in approximately the same location as in the corresponding slow and fast deceleration conditions of experiment 3 . This yielded a total of 12 stimuli. 


\subsection{Results}

Data were analysed with a 2 within (object A before, constant vs decelerating) $\times 2$ within (object A after, slow vs fast) $\times 3$ within (object B after, constant vs slow deceleration vs rapid deceleration) ANOVA. Means are reported in table 5. The main result of interest was moderately strong ratings of pulling at the slow speed of object A when the two objects remained in contact. This supports the interpretation proposed for the apparent lack of a pulling impression in experiment 3 , that the pulling impression is stronger at slow post-contact speeds.

Table 5. Mean ratings of pulling/pushing, experiment 4.

\begin{tabular}{lllrl}
\hline \multirow{2}{*}{ Object A before } & Object A after & \multicolumn{2}{l}{ Object B after } \\
\cline { 3 - 5 } & & constant & slow & rapid \\
\hline Constant & slow & -42.80 & -7.50 & 23.43 \\
& fast & -11.80 & 9.23 & 19.40 \\
Decelerating & slow & -44.10 & 12.07 & 25.47 \\
& fast & -2.27 & 22.47 & 32.37
\end{tabular}

Note. Ratings are on a scale from -100 (pulling) to +100 (pushing).

There was a significant main effect of object $\mathrm{A}$ after $\left(F_{1,29}=7.35, \mathrm{MSE}=3596.72\right.$, $p<0.05)$, with a higher mean at the fast speed (11.57) than at the slow speed $(-5.57)$. There was a significant main effect of object B after $\left(F_{2,58}=31.39, \mathrm{MSE}=2534.19, p<0.001\right)$. A posteriori paired comparisons with the Tukey test revealed the order rapid deceleration (25.17) $>$ slow deceleration $(9.07)>$ constant $(-25.24)$.

These two effects were qualified by a significant two-way interaction between them $\left(F_{2,58}=7.20, \mathrm{MSE}=1314.87, p<0.01\right)$. Simple effects analysis revealed a significant effect of object A after at constant speed of object B after $\left(F_{1,29}=10.54, \mathrm{MSE}=3773.97, p<0.01\right)$. This reflects the difference between object $B$ remaining in contact with object $A$ and not doing so, with a pulling impression occurring in the former (mean $=-43.45)$ and not in the latter $(-7.03)$. There was no significant effect of object A after at either slow deceleration of object $\mathrm{B}$ $\left(F_{1,29}=3.60, \mathrm{MSE}=1535.49\right)$ or rapid deceleration of object $\mathrm{B}\left(F_{1,29}=0.07, \mathrm{MSE}=917.01\right)$.

There were significant effects of object $B$ after at both values of object A after, and both of these conformed to the tendency in the main effect. The interaction reflects the fact that the range of means was greater at the slow speed of object A after than at the fast speed of object A after. This is likely to be a consequence of the difference made when the two objects remain in contact, which only happens at the slow speed of object A; at the slow speed of object A $\left(F_{2,58}=30.27, \mathrm{MSE}=2376.24, p<0.001\right)$; at the fast speed of object A after $\left(F_{2,58}=11.60, \mathrm{MSE}=1472.83, p<0.001\right)$. There were no other significant results.

\subsection{Discussion}

The two stimuli in which the objects remained in contact while moving to the left received substantially lower mean ratings than the other stimuli, -42.80 and -44.10 , as shown in table 5. These means indicate a moderate but consistent impression of pulling, and contrast with the lack of a pulling impression found in experiment 3 . This supports the hypothesis that the lack of a pulling impression in experiment 3 was due to the faster post-contact speed of the objects. It is also noteworthy that stimuli in which object B decelerated to a halt after contact gave rise to lower ratings in this experiment than in experiment 3 . It is likely that the converse explanation holds for pushing: the impression may be stronger at higher speeds. Michotte (1963) found that the launching effect was weaker at slow speeds of the causal object, and that tendency appears to be consistent with this. 


\section{General discussion}

In all forms of visual causal impression investigated hitherto, the object perceived as causal has been the one that moves first, and the object that is initially stationary is perceived as being acted on in some way by the initially moving object. This is the case for launching, entraining, pulling, and all other kinds of visual interaction impression (Michotte 1963; Scholl and Tremoulet 2000; White and Milne 1997, 1999, 2003). The present research therefore appears to be the first demonstration of a stimulus that gives rise to an impression that an object that only moves when it is contacted by another object is causal, and makes something happen to that other object. Experiments 1, 2, and 3 used different methods, with forced-choice verbal responses in experiment 1 , free verbal descriptions in experiment 2 , and a rating scale measure in experiment 3 ; and all three produced evidence for the occurrence of an impression in which object B pushes object A away in the separating motion stimuli. The only difference between the separating motion stimuli and the shared motion stimuli is that the two objects remained in contact in the latter but not in the former. If the two objects remain in contact, the pushing impression occurs only rarely (experiment 2). Under some circumstances, specifically slow post-contact motion, shared motion stimuli yield an impression that object A is pulling object B (experiment 4), but under other circumstances the stimuli appear ambiguous.

The findings from the first three experiments disconfirm the prior motion hypothesis, and the accounts by Michotte (1963) and Leslie (1995), in which prior motion of the object perceived as causal is necessary for the causal impression to occur. Michotte's account is strongly disconfirmed because prior motion of the object perceived as causal is a necessary condition for the Gestalt properties that are themselves necessary for a causal impression to occur. Leslie's account is basically a modular account, and the general principle of modules for causal perception could be preserved by postulating a different kind of module that interprets the motion pattern of the separating motion stimulus as causal. The findings do not fit, however, with Leslie's specific account in terms of forces exerted by moving objects. Of course, under many circumstances the object that moves first is perceived as causal; the launching effect is a case in point. The present findings do not disconfirm the importance of prior motion in stimuli such as launching effect stimuli. They only disconfirm the hypothesis that prior motion is necessary for the object in question to be perceived as causing the motion of another object. That proposition is central to the accounts of both Michotte (1963) and Leslie (1995), so the results show that a different approach to explaining visual impressions of causality is required. The findings are, however, consistent with the reasoning based on the matching hypothesis. The visual impression of pushing occurs because the stimulus can be unambiguously matched to stored representations of pushing actions, specifically pushing away an approaching object.

The results of experiment 3 indicated a stronger pushing impression if object $\mathrm{B}$ decelerated after contact than if it moved at a constant speed that was slower than object A's speed. The pushing impression was also stronger if object A moved at a slow but constant speed prior to contact. The optimal stimulus for the pushing impression is therefore likely to involve slow but constant speed of object A prior to contact, and fast initial speed followed by rapid deceleration of object $\mathrm{B}$ after contact, though the modest range of speeds used suggests that much remains to be learned about the effects of these variables.

So far, I have discussed the pushing impression as if it were a causal impression. An alternative view is that it is an impression of agentic motion, specifically an impression that a self-propelled object (object B in the separating motion stimuli) literally acts on object A to produce an outcome. This can be distinguished from visual impressions of physical causality on the grounds that the motion of object B is perceived as intentional, or as a product of inner mental states. The argument for this would be that no purely physical mechanism can operate 
in the way that object B operates in the separating motion stimuli, and that agency is the only plausible interpretation of object B's behaviour.

This is a complex issue. Under the present account, all visual impressions of causality, and all visual impressions of force, occur because kinematic information in visual input is matched to a stored representation, ultimately of an action on an object. The matching process generates the causal impression, because the stored representation provides what the visually available information does not provide. Actions on objects are the model for visual impressions of causality. This does not imply that all visual causal impressions are impressions of animate beings with mental states relevant to action, such as intentions, producing outcomes through internally generated motion. However, the motion of object A in a typical launching effect stimulus is not perceived as just inanimate motion. Michotte (1963) found that object A's motion gave rise to the impression that object A was approaching object B; that is, not just that object A was getting closer to object B, but that it was making for object B. The specificity of this impression can be ascertained by comparison with an experiment in which object A extended itself (ie stretched) in the direction of object B and object B moved off when the leading edge of object A contacted it (Michotte 1963, experiment 16). Observers reported the launching effect, but that it came about by chance, as if object A happened to encounter object B accidentally. This shows by contrast how object $\mathrm{A}$ is perceived as making for object $\mathrm{B}$ in the usual launching effect stimulus.

This impression of approaching is corroborated in research by Tremoulet and Feldman (2006). One object executed straight line motion that involved a single change of direction. The perceptual impression resulting from this stimulus varied depending on the position of a stationary dot in the frame. Different positions of the dot resulted in impressions that the moving object was making for the dot, avoiding it, or moving independently of it. It is not necessary to conclude that object A in the launching effect stimulus is literally perceived as animate, but its motion nevertheless fits with stored representations of actions by virtue of having no apparent cause and being directed at object B.

In the actions on objects account (White 2009), all visual causal impressions are ultimately derived from stored representations of actions on objects. This does not mean that they are perceived as actions in the sense that mental states such as intentions are perceived as giving rise to them. It does mean that there is a vestige of agency in the causal impression: the dividing line between visual impressions of physical causality and visual impressions of agency is not absolute and sharp. Michotte (1963) commented that observers of the typical launching stimulus often described it as if object A were an actor executing an action on object B. One or two participants in experiment 2 did the same for the separating motion stimuli. The attraction of such analogies stems directly from matching to stored representations of actions on objects. But there is no reason to think that object B in a separating motion stimulus is perceived any differently from object $\mathrm{A}$ in a typical launching stimulus. The distinction between perceived physical causality and perceived animate actions is not absolute because the former partake of some of the properties of the latter; but that does not mean that the objects involved in physical causality are perceived as animate beings with minds and intentions. It means only that our understanding of forces and causality in interactions between physical objects owes a good deal to our experience of our own actions on objects. Fundamentally, the visual causal impression that occurs in perception of these stimuli is an impression of a kind of interaction between objects, not an identification of an object as being of a particular kind.

If object B is perceived as moving of its own accord, could the findings for the separating motion stimulus be explained by models of perception of motion as animate? Several accounts of motion properties leading to perception of animate motion have been proposed (Biro and Leslie 2007; Csibra 2008; Dittrich and Lea 1994; Luo et al 2009; Rakison and Poulin-Dubois 2001; Rochat et al 1997; Southgate et al 2008; Tremoulet and Feldman 2000, 2006). None of these, however, has postulated or reported evidence for impressions of animate motion 
occurring for an object that starts to move when contacted by another object. Indeed, Rakison and Poulin-Dubois (2001) proposed that motion onset without contact from another object was a key criterion for identifying motion as animate. None of these accounts, therefore, can explain the present results.

Could it be argued that the impression that occurs with the separating motion stimuli is not a true visual causal impression in the way that the launching effect is? From my personal experience, I find this implausible. The causal impression that occurs with the separating motion stimulus is, for me, vivid and almost startling, and more compelling than the launching effect. This gives me no reason to think that the launching effect is in any way a more visual impression than the impression given by the separating motion stimulus is. Consensus on the pushing impression for stimuli in set 1 of experiment 1 was high, and a majority of participants in experiment 2 spontaneously produced descriptions of object B pushing object A. It is difficult to know how they could arrive at the kind of description, if they did not have a visual impression of causality with object B as the cause.

Another possible objection to the present results is that, in fact, the prior motion hypothesis is already known to be false. Imagine a ball bouncing on a floor. It could be argued that the floor causes the bounce of the ball even though the floor does not move, and that this is evidence of a visual causal impression occurring in the absence of prior motion, indeed any motion, of the causal object. There is evidence bearing on this argument. Michotte (1963, page 44, experiment 4) reported an experiment in which object A contacted object B as in a launching stimulus and then returned in the direction from which it came, while object B remained motionless. Michotte tried various different speeds, including those that had been found most favourable to the occurrence of the launching effect. He reported that "[n]o trace of a causal influence was ever found in this experiment ... there is no impression that the return movement is caused by the outward movement, nor even that it is brought about by contact with an obstacle" (page 44). He did report a character of activity, of striking, particularly when the speed of object A was high. In light of the study by White (2009), it is likely that this is an impression of force: object A is perceived as striking object B forcefully, and the amount of force increases as the speed of object A prior to contact increases. But this is distinct from the causal impression. The results for the shattering stimulus in experiment 2 confirm this view. In that stimulus object B did not move, and no participant produced a report of a causal impression with causality assigned to object B. So, contrary to the objection, there is no evidence that the prior motion hypothesis was already known to be false, because impressions of bouncing or rebounding or shattering are not causal impressions. ${ }^{(1)}$

A further possible objection is that participants might not have been reporting genuine perceptual impressions, but instead working out in higher cognition what sort of interaction was likely to have been taking place. Evidence for the launching effect has sometimes been criticised on similar grounds (Gemelli and Cappellini 1958; Joynson 1971), but improved methodology, brain imaging studies, and some ingenious experimental research have combined to establish beyond reasonable doubt that the launching effect is a genuine perceptual phenomenon (Blakemore et al 2001; Choi and Scholl 2004, 2006; Gordon et al 1990; Roser et al 2005; Scholl and Nakayama 2002, 2004).

In the present case, it is not clear that higher cognition would be much help in deciding what sort of interaction might have been going on. When presented with a separating motion stimulus, on what grounds could one deduce that object B pushed object A? This could

${ }^{(1)}$ Skeptical of Michotte's report, I constructed a rebound stimulus similar to the ones he used, and I was surprised to find myself concurring with his report: for me there was an impression of forceful activity on the part of object A, but no causal impression. An experimental investigation of this would be of some value to an understanding of the kinds of impression that occur when objects are perceived as interacting with each other. 
happen if object B was self-propelled, starting to move because of some internal energy source. But there is no cue from which to deduce that object B is self-propelled: it presents as an inert geometrical form. And its motion is triggered, if not actually elicited, by contact from object A, whereas a self-propelled object might be thought to move spontaneously. It could be argued that, in the separating motion stimuli, object $\mathrm{B}$ changed its motion characteristics by slowing to a halt, and that this could be a cue that object $B$ is the agent in the relation. In fact, object B underwent deceleration at constant rate, which is characteristic of the motion of an inanimate object encountering frictional forces, either air resistance or friction with the surface on which it is moving. If anything, object A should be viewed as animate because it does not slow down, as one would expect an inanimate moving object to do (Hubbard et al 2001). So object B's motion actually indicates lack of animacy more than object A's does, and this therefore cannot be used as a cue to identify object B as the agent. So inference in higher cognition does not suffice to specify choice of the pushing response.

The present findings disconfirm the prior motion hypothesis, but they do not disconfirm the requirement of temporal priority of the cause over the effect. Temporal priority means that a cause cannot occur after its effect in time, and observation of temporal priority was one of the criteria for identifying a causal relation postulated by Hume (1739/1978). Temporal priority means priority in time of the cause over the effect, not priority in time of motion of the causal object over motion of the effect object. With the separating motion stimulus, object B is perceived as pushing object A away. This perceptual impression involves an exertion of force by object $\mathrm{B}$ which produces the motion of object $\mathrm{A}$. The perceived exertion of force by object B is temporally prior to the outcome for object A. So the present findings disconfirm the prior motion hypothesis but say nothing about the temporal priority rule.

Under the present account, no visual causal impression will occur if the kinematic information in the stimulus is ambiguous, in the sense that it can be matched equally well to two different kinds of stored representation. This conflicts with a common observation that definite interpretations of ambiguous figures tend to be adopted by the visual system, but that the interpretation switches between the possible alternatives as the stimulus continues to be viewed. This is the case for the Necker cube, for example. This would seem to imply that different causal interpretations of ambiguous kinematic information should occur, and that the visual system should alternate between them. The main problem with investigating this possibility is that the stimuli are not static. The Necker cube, and other ambiguous figures, are presented as static images that can be scrutinised for prolonged periods, whereas the stimuli in the present research are not static and last for no more than $2.7 \mathrm{~s}$. This does not give much opportunity for a shift in perception to occur within a single presentation. However, the possibility of alternation in perceptual interpretations of ambiguous kinematic stimuli is not inconsistent with the present account, and would certainly be worthy of further investigation.

The theories discussed in this paper have been psychological theories about how kinematic information might be interpreted in terms of dynamics. An alternative approach comes from the domain of computational modelling. Mann et al (1997) developed a computational system that operates with an approximation to Newtonian mechanics, which it uses to generate dynamical interpretations of visual data, acquired through a camera. Examples include video sequences of a hand reaching for and lifting a coke can on a desk, and one car colliding with another and setting it in motion. The system can identify an object as active (if it is in motion at more than a certain speed) or passive (if it is not in motion). It can also use its Newtonian mechanics to derive possible dynamical interpretations of the visual sequence. These include the possibility that one or both objects have internal energy sources that power their movement. For the hand and coke can sequence, the system generates five physically plausible interpretations. A set of three preference rules is then used to order these in terms of likelihood. 
The main advantage of a model of this sort is it specifies quite precisely what is required for a dynamical interpretation of kinematic information to be made. This particular model, however, does not solve the problem presented by the separating motion stimuli. First, for the hand and coke sequence, the system identifies two of the five possible interpretations as equally favoured after the preference rules have been applied. In one of these, the hand has an internal motor and the coke can does not; in the other, the coke can has an internal motor and the hand does not. Thus, the system cannot discriminate between an interpretation in which the hand lifts the coke can and one in which the coke can lifts the hand. The same applies to the sequence involving a collision between two cars: the system cannot decide between an interpretation in which the first car pushes the second and one in which the second car pulls the first. In application to the stimuli used in the present research, the system would similarly be unable to distinguish between pushing and pulling interpretations, and therefore would be unable to predict the preference for the pushing interpretation with the separating motion stimulus and for the pulling interpretation with the shared motion stimulus. One reason for this inability is that the system does not integrate across time. Thus, in the reaching phase of the hand and coke can sequence, the system identifies the hand as active and the coke can as passive, but it does not draw on that information in the lifting phase. Of course, if it did, then it would identify the hand as causal. If applied to the separating motion stimulus, it would for the same reason identify object $A$ as causal: object $A$ and not object $\mathrm{B}$ is in motion prior to contact. This would be incorrect. Second, the preference rules are set by fiat of the researchers. One could change the way the system operates by changing the preference rules, and this is critical for generating predictions about causal perception, but it does not appear to be done in a principled way: one simply chooses the rules that yield plausible interpretations when applied. There does not appear to have been any further development of this system, but in principle computational modelling could have much to offer research in this area.

The present study opens up new possibilities for further research. Brain imaging studies have so far focussed on conventional launching effect stimuli. It is equally important to ascertain whether typical stimuli for the entraining and pulling impressions elicit similar patterns of brain activation; these have been inexplicably neglected. It would be important to show a similar pattern of brain activity for the separating motion stimuli. Research with young infants has also focussed almost entirely on the launching effect so far (though Bélanger and Desrochers 2001, reported a failure to find the entraining effect in infants aged 6 months), and for the same reasons research on pulling, entraining, and the separating motion stimuli with young infants would be critical to a full understanding of visual causal impressions.

Research on visual causal impressions has concentrated on the conventional launching stimulus almost to the exclusion of other forms of causal impression. It is not clear a priori that the launching stimulus is in any way more important or more fundamental than any other kind, only that it seems to conform to our preconceived ideas of what a causal relation should look like more than perhaps other kinds do. I hope the present study will break the mould of research in this area, and encourage researchers to investigate the occurrence of different kinds of causal impressions and the possibility that causal impressions may be found to occur in novel and unexpected places. This will facilitate progress to a fuller and more representative picture of the role that visual impressions of causality may play in the development of causal understanding as a whole.

Acknowledgments. The software used to construct and present the stimuli was created by Alan Milne. I am grateful to Inga Kristaponyte, Agnes Henson, and Carl Hodgetts for acting as experimenters, and to Lorraine Woods for assistance with the figures. 


\section{References}

Bélanger N D, Desrochers S, 2001 "Can 6-month-old infants process causality in different types of causal events?" British Journal of Developmental Psychology 19 11-21

Biro S, Leslie A M, 2007 “Infants' perception of goal-directed actions: development through cue-based bootstrapping" Developmental Science 10 379-398

Blakemore S-J, Fonlupt P, Pachot-Clouard M, Darmon C, Boyer P, Meltzoff A N, Segebarth C, Decety J, 2001 "How the brain perceives causality: an event-related fMRI study" NeuroReport 12 3741-3746

Choi H, Scholl B J, 2004 "Effects of grouping and attention on perception of causality" Perception \& Psychophysics 66 926-942

Choi H, Scholl B J, 2006 "Perceiving causality after the fact: postdiction in the temporal dynamics of causal perception" Perception 35 385-399

Cohen L B, Amsel G, 1998 "Precursors to infants' perception of causality" Infant Behavior and Development 21 713-731

Cohen L B, Amsel G, Redford M A, Casasola M, 1998 "The development of infant causal perception", in Perceptual Development: Visual, Auditory, and Speech Perception in Infancy Ed. A Slater (Hove, East Sussex: Psychology Press) pp 167-209

Cohen L B, Oakes L M, 1993 "How infants perceive simple causality" Developmental Psychology 29 $421-433$

Csibra G, 2008 "Goal attribution to inanimate agents by 6.5-month-old infants" Cognition 107 705-717

Dittrich W H, Lea S E G, 1994 "Visual perception of intentional motion" Perception 23 253-268

Fugelsang J A, Roser M E, Corballis P M, Gazzaniga M S, Dunbar K N, 2005 "Brain mechanisms underlying perceived causality" Cognitive Brain Research 24 41-47

Gemelli A, Cappellini A, 1958 “The influence of the subject's attitude in perception” Acta Psychologica $1412-23$

Gordon I E, Day R H, Stecher E J, 1990 "Perceived causality occurs with stroboscopic movement of one or both stimulus elements" Perception 19 17-20

Hubbard T L, Blessum J A, Ruppel S E, 2001 “Representational momentum and Michotte's (1946/1963) 'launching effect' paradigm" Journal of Experimental Psychology: Learning, Memory, and Cognition 27 294-301

Hume D, 1978 A Treatise of Human Nature (Oxford: Oxford University Press; original work published in 1739)

Joynson R B, 1971 "Michotte's experimental methods" British Journal of Psychology 62 293-302

Leslie A M, 1995 “A theory of agency”, in Causal Cognition: A Multidisciplinary Debate Eds D Sperber, D Premack, A J Premack (New York and Oxford: Oxford University Press) pp 121-141

Leslie A M, Keeble S, 1987 "Do six-month-old infants perceive causality?" Cognition 25 265-288

Luo Y, Kaufman L, Baillargeon R, 2009 "Young infants' reasoning about physical events involving inert and self-propelled objects" Cognitive Psychology 58 441-486

Mann R, Jepson A, Siskind J M, 1997 "The computational perception of scene dynamics" Computer Vision and Image Understanding $\mathbf{6 5} 113-128$

Michotte A, 1963 The Perception of Causality (New York: Basic Books, originally published 1946)

Muentener P, Carey S, 2010 "Infants' causal representations of state change events" Cognitive Psychology 61 63-86

Natsoulas T, 1961 "Principles of momentum and kinetic energy in the perception of causality" American Journal of Psychology 74 394-402

Newman G E, Choi H, Wynn K, Scholl B J, 2008 "The origins of causal perception: evidence from postdictive processing in infancy" Cognitive Psychology 57 262-291

Oakes L M, 1994 "Development of infants' use of continuity cues in their perception of causality" Developmental Psychology 30 869-879

Oakes L M, Cohen L B, 1990 "Infant perception of a causal event" Cognitive Development 5 193-207

Rakison D H, Poulin-Dubois D, 2001 "Developmental origin of the animate-inanimate distinction" Psychological Bulletin 127 209-228

Rochat P, Morgan R, Carpenter M, 1997 "Young infants' sensitivity to movement information specifying social causality” Cognitive Development 12 537-561 
Roser M E, Fugelsang J A, Dunbar K N, Corballis P M, Gazzaniga M S, 2005 "Dissociating processes supporting causal perception and causal inference in the brain" Neuropsychology 19 591-602

Saxe R, Carey S, 2006 "The perception of causality in infancy" Acta Psychologica 123 144-165

Schlottmann A, Ray E, Mitchell A, Demetriou N, 2006 "Perceived social and physical causality in animated motions: spontaneous reports and ratings" Acta Psychologica 123 112-143

Scholl B J, Nakayama K, 2002 "Causal capture: contextual effects on the perception of collision events” Psychological Science 13 493-498

Scholl B J, Nakayama K, 2004 "Illusory causal crescents: misperceived spatial relations due to perceived causality" Perception 33 455-469

Scholl B J, Tremoulet P D, 2000 "Perceptual causality and animacy" Trends in Cognitive Sciences 4 299-309

Southgate V, Johnson M H, Csibra G, 2008 "Infants attribute goals even to biomechanically impossible actions" Cognition 107 1059-1069

Tremoulet P D, Feldman J, 2000 "Perception of animacy from the motion of a single object" Perception 29 943-951

Tremoulet P D, Feldman J, 2006 "The influence of spatial context and the role of intentionality in the interpretation of animacy from motion" Perception \& Psychophysics 68 1047-1058

White P A, 2005 "Visual impressions of interactions between objects when the causal object does not move" Perception 34 491-500

White P A, 2006 "The causal asymmetry" Psychological Review 113 132-147

White P A, 2007 "Impressions of force in visual perception of collision events: a test of the causal asymmetry hypothesis" Psychonomic Bulletin \& Review 14 647-652

White P A, 2009 "Perception of forces exerted by objects in collision events" Psychological Review $116580-601$

White P A, 2010 "The property transmission hypothesis: a possible explanation for visual impressions of pulling and other kinds of phenomenal causality" Perception 39 1240-1253

White P A, 2012a "Visual impressions of causality: effects of manipulating the direction of the target object's motion in a collision event" Visual Cognition 20 121-142

White P A, 2012b, "The experience of force: the role of haptic experience of forces in visual perception of object motion and interactions, mental simulation, and motion-related judgments" Psychological Bulletin 138 589-615

White P A, Milne A, 1997 "Phenomenal causality: impressions of pulling in the visual perception of objects in motion" American Journal of Psychology 110 573-602

White P A, Milne A, 1999 "Impressions of enforced disintegration and bursting in the visual perception of collision events" Journal of Experimental Psychology: General 128 499-516

White P A, Milne A, 2003 "Visual impressions of penetration in the perception of objects in motion" Visual Cognition 10 605-619

Yela M, 1952 "Phenomenal causation at a distance" Quarterly Journal of Experimental Psychology 4 139-154 